\title{
New Phase Unwrapping Strategy for Rapid and Dense 3D Data Acquisition in Structured Light Approach
}

\author{
G Sai Siva and L Kameswara Rao \\ Department of Instrumentation \\ Indian Institute of Science \\ Bangalore-12, India.
}

\begin{abstract}
Sinusoidal structured light projection (SSLP) technique, specifically- phase stepping method, is in widespread use to obtain accurate, dense 3-D data. But, if the object under investigation possesses surface discontinuities, phase unwrapping (an intermediate step in SSLP) stage mandatorily require several additional images, of the object with projected fringes (of different spatial frequencies), as input to generate a reliable 3D shape. On the other hand, Color-coded structured light projection (CSLP) technique is known to require a single image as in put, but generates sparse 3D data. Thus we propose the use of CSLP in conjunction with SSLP to obtain dense 3D data with minimum number of images as input. This approach is shown to be significantly faster and reliable than temporal phase unwrapping procedure that uses a complete exponential sequence. For example, if a measurement with the accuracy obtained by interrogating the object with 32 fringes in the projected pattern is carried out with both the methods, new strategy proposed requires only 5 frames as compared to 24 frames required by the later method.
\end{abstract}

Keywords: Structured light projection, shape measurement; phase stepping; phase unwrapping; color-coding; surface discontinuities

\section{Introduction}

The measurement of surface shape by use of projected structured light patterns is a well-developed technique. Especially, SSLP techniques have been extensively used as it can give accurate and dens 3D data. The procedure involves- projecting a pattern on to the object from an offset angle and recording the image of the pattern which is phase modulated by the topographical variations of the object surface. An automated analysis is then carried out to extract the phase from the deformed fringe pattern mostly using either FFT[1] or phase stepping[2] methods, both of them 
produce wrapped phase distribution. The reconstruction of surface profile of objects with inherent surface discontinuities or having spatially isolated regions is usually a difficult problem by using standard phase unwrapping techniques.

To overcome this problem several phase unwrapping strategies were developed [3][4][5]. All of them mandataroly require multiple phase maps generated by varying the spatial frequency of projected fringe pattern either linearly or exponentially. Further, the degree of reliability varies from method to method.

A different class of structured light projection techniques relies upon color-coded projection. They are capable of extracting 3D data from a single image. Different color-coding strategies can be seen in [6],[7]. However, they can give only sparse 3D data. In the following sections we suggest an approach for obtaining dens 3D data of objects even with surface discontinuities while requiring minimum number of input images as compared to any of the contemporary phase unwrapping algorithms

\section{Method}

The first step of profiling objects in the proposed method involves the generation of wrapped phase map using "four-frame phase shifting algorithm". The fundamental concepts of phase stepping method, is described elsewhere [2], is only briefly reviewed here.

\subsection{Phase Stepping Algorithm}

When a sinusoidal fringe pattern is projected on to a 3-D diffuse object, the mathematical representation of the deformed fringe pattern may be expressed in the general form

$I(x, y)=a(x, y)+b(x, y) \cos \phi(x, y)$

Where $\mathrm{a}(\mathrm{x}, \mathrm{y}), \mathrm{b}(\mathrm{x}, \mathrm{y})$ represent unwanted irradiance variations arising from the non-uniform light reflection by a test object. The phase function $\phi(\mathrm{x}, \mathrm{y})$ characterizes the fringe deformation and is related to the object shape $\mathrm{h}(\mathrm{x}, \mathrm{y})$. The principal task is to obtain $\phi(\mathrm{x}, \mathrm{y})$ from the measured fringe-pattern intensity distribution. Upon shifting the original projected fringe pattern by a fraction $1 / \mathrm{N}$ of its period $\mathrm{P}$, the phase of the pattern represented by Eq.(1) is shifted by $2 \pi / \mathrm{N}$. Using four images $\phi(x, y)$ can be retrieved independently of the other parameters in Eq.(1):

$\phi(x, y)=\arctan \left[\left(I_{4}-I_{2}\right) /\left(I_{1}-I_{3}\right)\right]$ 


\subsection{Phase Unwrapping}

The object phase calculated according to Eq.(2) is wrapped in the range $-\pi$ to $\pi$. The true phase of the object is

$\phi_{\text {un }}(\mathrm{x}, \mathrm{y})=\phi(\mathrm{x}, \mathrm{y})+2 \mathrm{n}(\mathrm{x}, \mathrm{y}) \pi$

where $n(x, y)$ is an integer. Unwrapping is only a process of determining $\mathrm{n}(\mathrm{x}, \mathrm{y})$. A conventional spatial phase unwrapping algorithm search for locations of phase jumps in the wrapped phase distribution and adds/subtracts $2 \pi$ to bring the relative phase between two neighboring pixels into the range of $-\pi$ to $\pi$. Thus irrespective of actual value of $n(x, y)$ to be evaluated, they always assign \pm 1 thereby fails to reliably unwrapp phase maps in profiling objects with surface discontinuities. In order to determine $\mathrm{n}(\mathrm{x}, \mathrm{y})$ we are introducing the following procedure:

\subsection{New Unwrapping Procedure}

In this new approach, an additional image of the object captured under illumination of a color-coded pattern is used for calculating $n(x, y)$. A color-coded pattern is generated using MATLAB in a computer and projected with the help of a LCD projector. Color-coded pattern generated comprises of: an array of rectangular shaped bands, with each band identified uniquely by its color and arranged in a specific sequence as shown in Fig.1. This pattern is projected onto the reference plane and its image $\left(\mathrm{C}_{\mathrm{r}}(\mathrm{i}, \mathrm{j})\right)$ is recorded. A non-planar real object distorts the projected structured pattern in accordance with its topographical variations $\left(\mathrm{C}_{0}(\mathrm{i}, \mathrm{j})\right)$. Now, we know a priori the color expected to be returned by every point from an ideally planar object surface. Deviations in the detected color at any point on the non-planar object surface essentially correspond to local height deviations. Therefore, from the knowledge of observed color $\left(\mathrm{C}_{\mathrm{o}}\right)$ and expected color $\left(\mathrm{C}_{\mathrm{r}}\right)$, height deviation at every point on object surface can be expressed in terms of difference of their band indices (m) as explained in [8]. If the width of every band (w) is made equal to the pitch of the gray-scale fringe pattern used in phase stepping, then $\mathrm{m}$ can be directly related to $\mathrm{n}(\mathrm{x}, \mathrm{y})$ in the Eq.(3). This is the basis for determining $\mathrm{n}(\mathrm{x}, \mathrm{y})$ unambiguously. Procedure to extract necessary information from the deformed color-coded pattern and exploiting its use in determining $\mathrm{n}(\mathrm{x}$, y) is presented in [8].

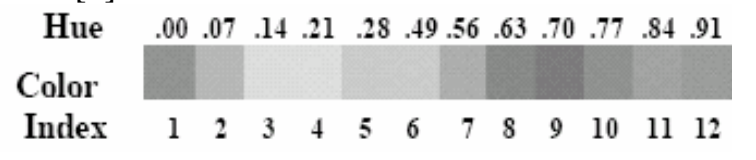

Fig.1 Generated color-coded pattern 


\section{Experimental Results}
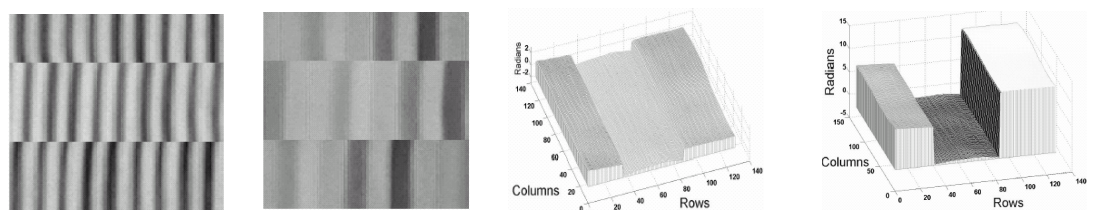

Fig.2 (a) Fringe pattern (b) color-coded pattern on the surface of an object with two step discontinuities (c) wrapped phase map obtained with phase stepping (d) phase map after unwrapping with the help of Fig.2 (b)

It is impossible to unwrap the phase map in Fig.2(a) correctly by conventional spatial methods because the phase jumps at the steps are too large (more than $2 \pi$ ). Even though it is impossible to determine exact numbers of fringes shifted at each step height from Gray scale fringe pattern (Fig. 2(a)) alone, color-coded pattern on the object surface clearly reveals this information as can be seen from Fig. 2(b).

\section{Conclusions}

The new approach proposed, that combines CSLP and SSLP in a specific way, has resulted in a new and more powerful method for generating rapid and dense 3D data. It is shown to be significantly faster and reliable than temporal phase unwrapping that uses complete exponential sequence and compared to it the reduction both in image acquisition and in analysis times by the factor $\left[N^{*}\left(\log _{2} \mathrm{~S}+1\right)\right] /(\mathrm{N}+1)$ is an important advantage of the present approach.

$(\mathrm{N}$ : number of frames used in phase stepping and $\mathrm{S}$ : number of fringes in the pattern projected).

\section{References}

[1] Mitsuo Takeda et al., (1982) J.Opt. Soc. America 72(1): 156-160

[2] V.Srinivasan et al., (1984) Applied Optics 23(18): 3105-3108

[3] H.Zhao et al., (1994) Applied Optics 33: 4497-4500

[4] J.M. Huntley and H.O. Saldner, (1997) Meas. Sci. Technol 8: 986-992

[5] Hong Zhang et al., (1999) Applied Optics 38(16), 3534-3541

[6] Weiyi Liu et al., (2000) Applied Optics 39(20), 3504-3508

[7] Li Zhang et al., (2002) Proc. of the 1st Int. Symp. on 3DPVT, 24-36

[8] Sai Siva and L K Rao, (2005) Proc.of SPIE, vol:5856, Paper:78. 